\title{
Levels of a Hydrojuglone Glucoside in Developing Pecan Leaves in Relation to Scab Susceptibility
}

\author{
R.C. Gueldner and I.E. Yates \\ Russell Research Center, Agricultural Research Service, U.S. Department of Agriculture, Athens, \\ GA 30613 \\ C.C. Reilly and B.W. Wood \\ Southeastern Fruit and Tree Nut Laboratory, Agricultural Research Service, U.S. Department of \\ Agriculture, Byron, GA 31008 \\ M.T. Smith \\ Jamie Whitten Delta States Research Center, Agricultural Research Service, U.S. Department of \\ Agriculture, Stoneville, MS 38776
}

Additional index words. Carya illinoensis, Cladosporium caryigenum, juglone, maturation, infection

\begin{abstract}
Polyphenols were analyzed in expanding buds and developing leaves of pecan [Carya illinoensis (Wangenh.) C. Koch] cultivars with varying responses to Cladosporium caryigenum (Ell. et Lang. Gottwald), the organism causing scab. Plant tissue extracts were examined by high-performance liquid chromatography using a water : methanol gradient to separate polyphenolic components on a C-18 reversed phase column. A diode-array detector was used to identify profile components by retention times and computer matching of ultraviolet spectra to standard compounds in a library. Concentrations of these polyphenols were compared throughout the growing season in leaves of pecan cultivars with low ('Elliott'), intermediate ('Stuart'), and high ('Wichita') susceptibility to scab; during susceptibility to infection by Cladosporium caryigenum from 16 cultivars; and in 'Wichita' leaf discs with and without scab lesions. The major polyphenolic constituent of tissues for all cultivars was identified as hydrojuglone glucoside, which was detected in intact buds and leaves throughout the growing season. Hydrojuglone glucoside concentration increased concomitantly with leaf expansion and then declined slowly. Juglone was barely, if at all, detectable, regardless of leaf age. No correlation was found between cultivar susceptibility to pecan scab and the levels of either juglone or hydrojuglone glucoside in the healthy leaves of 16 cultivars. Leaf tissue with scab lesions had significantly higher juglone and hydrojuglone glucoside levels than leaf discs without scab lesions. Chemical names used: 4-8-dihydroxy-1-naphthyl в-D-glucopyranoside (hydrojuglone glucoside); 1,5-hydroxy-naphthoquinone (juglone).
\end{abstract}

Pecan, a native North American plant species of the hickory (Carya) genus, has developed over the last hundred years into a valuable commercial nut industry (Wood et al., 1990). Since the beginning of this century (Nolen, 1926; Latham and Rushing, 1988), pecan scab caused by Cladosporium caryigenum (Ell. et Lang. Gottwald) has been the major disease limiting pecan production in humid climates. Scab susceptibility among pecan cultivars and the development of resistance as leaves age, even in susceptible cultivars, has been known for a long time. Leaves of susceptible cultivars are subject to infection when less than $\approx 28$ days old (Demaree, 1924).

Scab can be controlled by applying appropriate fungicides and related management strategies (Ellis et al., 1991). However, excessive rains may create orchard conditions that prevent the use of the machinery needed for spray application. Furthermore, some of the most effective fungicides are in jeopardy of losing their registration by the U.S. Environmental Protection Agency for use on pecan. The threatened loss of registration, growers' needs to reduce crop-production costs, and perceived adverse effects of fungicides on the environment have created a renewed interest in

Received for publication 22 April 1993. Accepted for publication 19 July 1993 . The authors gratefully acknowledge the leaf evaluation for fungal damage by K. Cason, the statistical treatment of the level of hydrojuglone glucoside by D. Sparks, and the technical assistance of J. Lambert, R. Martin, and D. Maxey. Trade names are used in this publication to provide specific information. Mention of a trade name does not constitute a guarantee or warranty of the product or an endorsement by the USDA over other products not mentioned. The cost of publishing this paper was defrayed in part by the payment of page charges. Under postal regulations, this paper therefore must be hereby marked advertisement solely to indicate this fact. developing alternative control methods.

One way to depend less on fungicides for scab control is to use genetically based resistance of certain pecan cultivars (Thompson and Grauke, 1990). Genetic variance has scarcely been used to develop resistance to pecan pests, which are primarily fungal diseases and insects. An apparently diverse range of field reactions to scab disease exists among the numerous native and escaped seedlings, as well as in the many acceptable cultivars grown in the southeastern United States. Leaves and fruits on 'Wichita' trees can be totally destroyed from pecan scab, whereas adjacent 'Elliott' trees do not develop disease symptoms. The mechanism of this variance in disease reaction has not been established.

The utility of genetically based resistance to scab can be applied only with a greater understanding of the mechanism of action. Juglone, a polyphenol, has long been recognized as the signature metabolite of the Juglandaceae and as possessing toxic properties (Bell, 1981). In vitro toxicity of juglone against Cladosporium caryigenum and compounds identified in leaf and fruit extracts from selected pecan cultivars and other Carya sp. by thin-layer chromatography (TLC) have led to the proposal that elevated juglone levels resulted in resistance to infection by Cladosporium caryigenum (Hedin et al., 1979; Langhans et al., 1978). However, Wood et al. (1988) proposed that factors other than juglone likely were involved in resistance of pecan foliage and nuts to scab. The objective of this study was to analyze the polyphenolic composition of pecan buds and leaves with regard to developmental stage and cultivar susceptibility to scab. In addition, leaf tissue with and without scab damage from susceptible 'Wichita' was evaluated for polyphenolic content.

J. Amer. Soc. Hort. ScI. 119(3):498-504. 1994 


\section{Materials and Methods}

Plant material. Buds or leaves from the first cycle of shoot growth were collected from mature trees maintained by recommended orchard practices, but without chemical sprays, at the Southeastern Fruit and Tree Nut Laboratory in Byron, Ga. Four trees of each of the 16 cultivars, ranging from low to high scab susceptibility, were selected for sampling in early April. Samples were taken weekly from each tree from 3 Apr. to 21 June and at approximate monthly intervals thereafter. Cultivar scab ratings were derived from published data (Anonymous, 1970; Romberg, 1931; Sanderlin, 1987; Sherman and Gammon, 1977) recorded by Sparks (1992). Reported values were converted to a scale of 1 to 4 (low to high susceptibility, respectively) and the average for each cultivar was used as the value for cultivar susceptibility. Ratings from published data concurred with the authors' field observations over a 20-year period, with one exception. One report of 'Barton' as having high susceptibility to scab (Anonymous, 1970) was discounted because the authors have never observed scab lesions on it. No previously published rating for 'Pawnee' exists; thus, susceptibility is based on personal observations (authors, D. Sparks).

Collections were taken around each tree from the northern, eastern, southern, and western sides. Sampling was designed to ensure exhaustive analyses throughout the life history of the pecan leaf and from cultivars with varying responses to scab. Plant material used in this study had no macroscopically visible evidence of either disease, insect, or mechanical injury. Undamaged tissue was selected for the analyses of in vivo polyphenols of cultivars with gradients in scab susceptibility. Inherent polyphenolic composition could have been altered by general injury responses as described in the discussion. The collected tissue varied with the progression of shoot development (Fig. 1). Samples taken before budbreak (Fig. 1A) or at budbreak (Fig. 1B) were excised from the second or third subterminal bud from the apex of the 1-year-old shoot. Tissue taken before budbreak was the intact bud with the inner-bud scale, and tissue taken at budbreak included the entire bud without attending scales. Once the current year's shoot began to elongate, the second leaflets from the leaf base were taken from the second leaf from the base of the developing shoot (Fig. 1C). Thereafter, all cultivars were sampled from the third leaflet (Fig. 1D) from the leaf base on the fourth leaf from the shoot base.

Leaf extractions and high performance liquid chromatograpphy (HPLC) analysis. Tissue $(\approx 1 \mathrm{~g} / 10 \mathrm{ml}$ methanol containing $1 \mathrm{mg}$ of chrysin) was macerated with a homogenizer (Brinkman, Norwalk, Conn.) immediately after collection. Chrysin served as the internal standard for quantification of juglone and hydrojuglone to equalize slight differences in weight of tissue extracted among samples. The extracts were filtered through $1 \mathrm{~cm}^{2}$ cellulose tissue packed into a 6-mm i.d. borosilicate glass disposable pipette and the eluent was analyzed at $210 \mathrm{~nm}$ by HPLC (model 1090, Hewlett Packard, Sunnyvale, Calif.). Samples were analyzed on a reversed phase C-18 (octadecylsilane) column $(4.6 \mathrm{~mm} \times 25 \mathrm{~cm}$ with a $5-\mu$ particle diameter) (Alltech, Deerfield, Ill.), using a gradient of 5\% to $100 \%$ methanol containing $0.1 \%$ phosphoric acid.

Selected profile components were identified with a diode-array detector by computer matching of ultraviolet (UV) spectra with known standards of catechin, juglone, and hydrojuglone glucoside stored in the computer library. Catechin and juglone were purchased from Aldrich (Milwaukee, Wis.). 'Mahan' pecan leaves were the source for hydrojuglone glucoside. Extraction of 'Mahan' pecan leaves in methanol as described above and subsequent C-18 preparative chromatography yielded crystalline hydrojuglone glu-
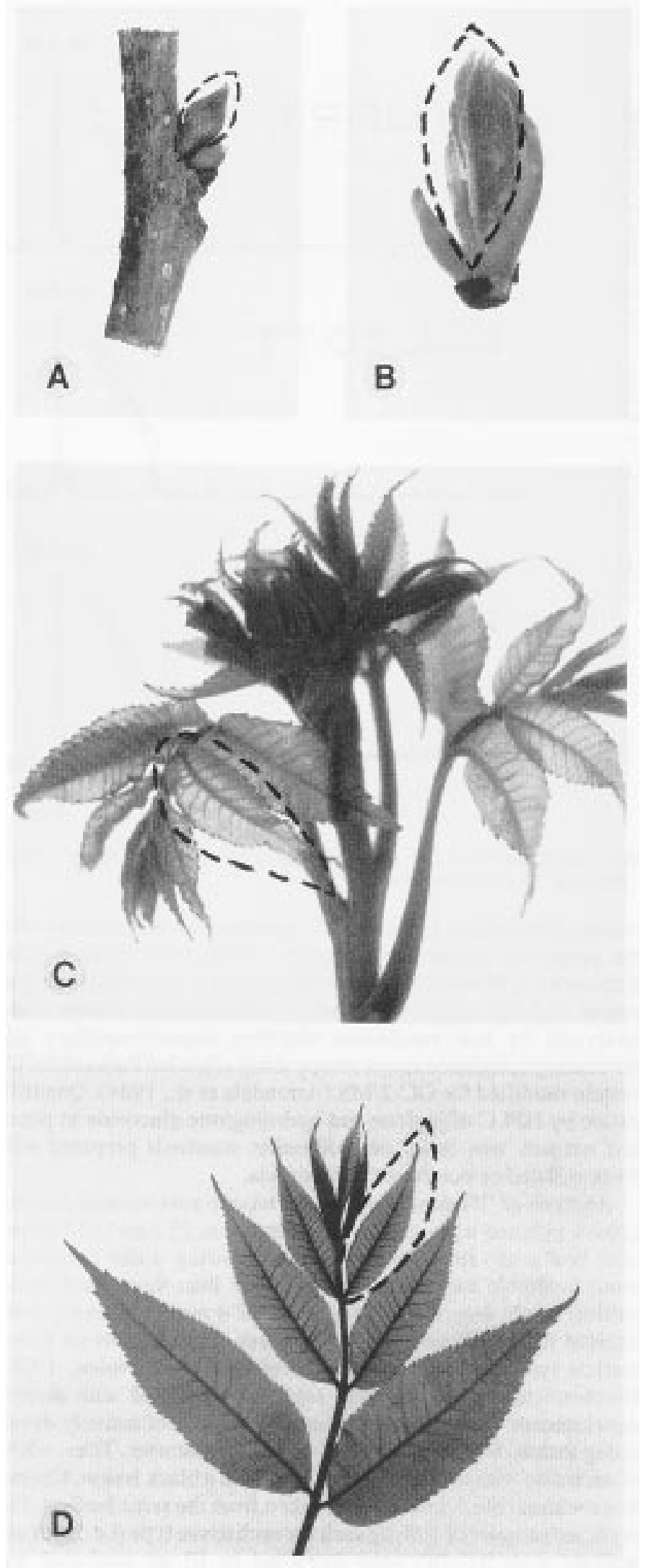

Fig. 1. Development of tissues sampled (enclosed by dashed lines) for 'Stuart' pecan. (A) Pre-budbreak. Intact bud collected 9 Apr. (B) Budbreak with green leaf tissue visible. Tissue interior to bud scale collected 16 Apr. (C) Early shoot and leaf petiole elongation. Second leaflet from base on second leaf collected 23 Apr. (D) Leaf expansion. Third leaflet from base on fourth leaf collected $30 \mathrm{Apr}$. to 7 Oct. 


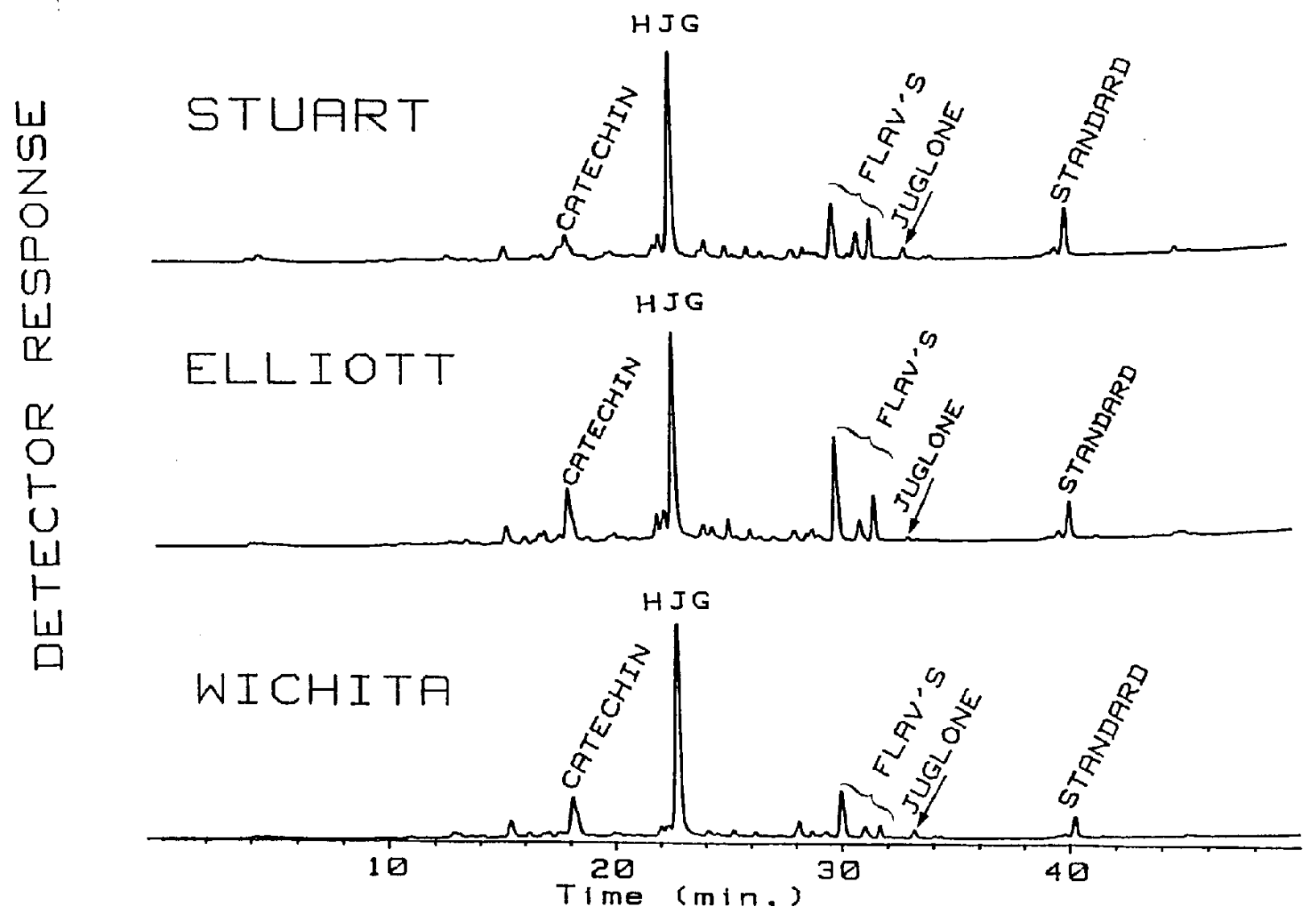

Fig. 2. HPLC profiles at $210 \mathrm{~nm}$ of polyphenols extracted from 'Stuart', 'Elliott', and 'Wichita' leaves collected 21 May. Abbreviations used: HJG = hydrojuglone glucoside, FLAV'S = flavonoids.

coside. The melting point and UV spectrum were consistent with the properties reported by Daglish (1950) for a hydrojuglone glucoside. A trimethylsilyl ether derivative of hydrojuglone glucoside had the required number of trimethylsilyl groups when analyzed by low resolution electron impact/capillary gas chromotography/mass spectroscopy using a Hewlett Packard 5958B system modified for GC-2/MS (Arrendale et al., 1984). Quantification by HPLC of juglone and hydrojuglone glucoside in pecan leaf extracts was based on calibration standards prepared with these isolated or purchased compounds.

Analysis of 'Wichita' leaf tissue with and without scab lesions. Leaves infected with scab were collected on 25 June $(\approx 14$ weeks after budbreak) from 'Wichita' trees growing under conditions more favorable for disease development than those used in the cultivar study described above. Discs of $4 \mathrm{~mm}$ in diameter were excised from leaflets with a cork borer. Scab lesions on pecan leaflets typically range from a speck to $6 \mathrm{~mm}$ (Nolen, 1926). Biochemical changes in plant material associated with disease development were expected to occur in regions of actively developing lesions which usually are $<2 \mathrm{~mm}$ in diameter. Thus, $\approx 50 \%$ of each disc with disease was covered with a black lesion. Control discs without black lesions were taken from the same leaflets. Ten replicate samples of $100 \mathrm{mg}$ each for each tissue type (i.e., with and without scab lesions) were extracted in $10 \mathrm{ml}$ methanol and analyzed as described above.

Statistical analyses. Data on the seasonal abundance of hydrojuglone glucoside in 'Elliott', 'Stuart', and 'Wichita' pecan were subjected to regression analysis with localized polynomial smoothing. Increase in early season was derived from data at peak concentration (week 3 for 'Stuart' and 'Wichita' and week 4 for
'Elliott') and the 3 weeks before. The decrease was derived from data at peak concentration and all subsequent time points. Statistical differences among cultivars for relative level of hydrojuglone glucoside at 2, 4, and 8 weeks after budbreak were determined by analysis of variance (ANOVA) and significant means were separated by Duncan's multiple range test. Quantities of hydrojuglone glucoside in 'Wichita' leaf tissue with and without scab lesions were compared by the unpaired $t$ test (Gomez and Gomez, 1984).

\section{Results}

Gross morphological features used to characterize shoot development. Gross morphological features of the buds and shoots (Fig. 1) were used to gauge each cultivar's development. Markers included bud enclosure by inner bud scale for pre-budbreak (Fig. 1A), green tissue visible at the bud apex for budbreak (Fig. 1B), leaves spreading away from shoot axis for commencement of shoot and leaf petiole elongation (Fig. 1C), and leaflets unfolding for leaf expansion (Fig. 1D). Temporal development for 'Stuart' (Fig. 1) was characteristic for all cultivars except for 'Desirable', 'Elliott', and 'Moore', in which budbreak preceded the other 13 cultivars by $\approx 1$ week.

Polyphenolic composition of pecan leaves. A methanol gradient of 5\%, 30\%, 50\%, and $100 \%$ applied to the HPLC reversed phase $\mathrm{C}-18$ column at $0,15,25$, and $40 \mathrm{~min}$, respectively, resulted in elution of all polyphenolic constituents from pecan leaf extracts before 35 min (Fig. 2). HPLC profiles of 'Stuart', 'Elliott', and 'Wichita' with intermediate, resistant, and susceptible scab reactions, respectively, were qualitatively similar. Polyphenolic constituents of these representative cultivars are demonstrated with 
HPLC profiles from when juglone first was detected in 'Elliott' pecan leaves (21 May). Identified constituents, in order of elution time, included catechin, hydrojuglone glucoside, flavonoids, and juglone. The most prominent constituent by far was hydrojuglone glucoside, with catechin, flavonoids, and juglone present in much lower concentrations. Catechin eluted at the relative rate indicated in Fig. 2, but was not clearly resolved from an unidentified compound with nearly the same retention time. Co-elution of the unidentified compound with catechin was indicated by the shoulder detected on the catechin peak. Catechin quantification was not attempted as a result of the co-elution of catechin and the uniden-

Table 1. Juglone concentration ( $\%$ leaf fresh weight $) \times 10^{-3}$ and comparison to hydrojuglone concentration in leaves of 16 pecan cultivars during the period of scab susceptibility.

\begin{tabular}{|c|c|c|c|c|c|c|}
\hline \multirow[b]{2}{*}{ Cultivar } & \multirow{2}{*}{$\begin{array}{l}\text { Scab } \\
\text { rating }^{2}\end{array}$} & \multicolumn{4}{|c|}{ Weeks after budbreak } & \multirow[b]{2}{*}{ HJG: $J^{y}$} \\
\hline & & 1 & 2 & 3 & 4 & \\
\hline Barton & 1.00 & $--^{x}$ & --- & --- & 0.7 & 1800:1 \\
\hline Elliott & 1.00 & --- & --- & --- & --- & $\mathrm{nd}^{\mathrm{w}}$ \\
\hline Sumner & 1.00 & ---- & 2.4 & 5.4 & --- & $300: 1$ \\
\hline Kiowa & 1.00 & --- & --- & 0.5 & --- & 6900:1 \\
\hline Farley & 1.55 & --- & --- & 0.9 & 0.7 & $400: 1$ \\
\hline Stuart & 1.95 & --- & 1.6 & --- & --- & 1600:1 \\
\hline Pawnee & 2.00 & --- & --- & 0.3 & --- & $2700: 1$ \\
\hline Curtis & 2.10 & --- & --- & --- & 0.7 & 2800:1 \\
\hline Cape Fear & 2.20 & --- & --- & --- & --- & nd \\
\hline Moore & 2.65 & --- & --- & 0.7 & 0.6 & 2000:1 \\
\hline Cheyenne & 2.85 & --- & --- & 4.1 & 4.9 & $500: 1$ \\
\hline Desirable & 2.96 & --- & --- & 0.5 & 1.5 & $700: 1$ \\
\hline Schley & 2.98 & --- & --- & 1.6 & 47.5 & $200: 1$ \\
\hline Western Schley & 3.70 & 1.9 & 2.5 & 25.5 & 10.8 & $100: 1$ \\
\hline Cherokee & 3.85 & --- & 0.4 & 2.4 & 47.6 & $100: 1$ \\
\hline Wichita & 4.00 & --- & 17.3 & 7.0 & 9.6 & $700: 1$ \\
\hline
\end{tabular}

${ }^{\mathrm{z}} 1$ = low susceptibility, 4 = high susceptibility.

${ }^{y}$ Ratio of the concentration hydrojuglone glucoside to juglone.

xuglone was not detected.

${ }^{\mathrm{w}} \mathrm{A}$ value was not determined as juglone was not detected. tified component.

Abundance of juglone and hydrojuglone glucoside during the growing season. Juglone was not a consistently detectable polyphenolic constituent of pecan leaves, either among the 16 cultivars or within the same cultivar either during or after the scab susceptibility period (Tables 1 and 2). Juglone was absent in all cultivars at both pre-budbreak and budbreak and was detected only in 'Western Schley' 1 week after budbreak (Table 1). During the first 4 weeks after budbreak, juglone was not detected in the leaves of either 'Elliott' or 'Cape Fear', despite that 'Elliot' is one of the least scab-susceptible pecan cultivars. 'Western Schley' was the only cultivar with detectable juglone in each of the 4 weeks after budbreak. Juglone was detected 2 weeks after budbreak in 'Sumner', 'Stuart', 'Western Schley', 'Cherokee', and 'Wichita' and 3 weeks after budbreak in all cultivars except for 'Barton', 'Elliott', 'Stuart', 'Curtis', and 'Cape Fear'. 'Barton' and 'Curtis' first had detectable juglone levels 4 weeks after budbreak. Although it was detected in 3-week-old leaves of 'Sumner', 'Kiowa', and 'Pawnee', juglone was not present in these cultivars at 4 weeks.

Juglone detection varied during the weeks of leaf scab susceptibility, as well as for the rest of the growing season (Table 2). Juglone was detected on all sampling dates in only 'Sumner', 'Western Schley', and 'Cherokee'. In all other cultivars, juglone was not detected from one ('Barton', 'Kiowa', 'Cape Fear', 'Cheyenne', 'Schley', and 'Wichita') up to seven ('Pawnee') sampling times.

Hydrojuglone glucoside, which was the major polyphenolic constituent in all tissues, was present from the first collection time of intact buds in early April until leaf senescence in October in all 16 cultivars. The hydrojuglone glucoside concentration was at least one hundred times that of juglone for all cultivars during the scab susceptibility period (Table 1). The hydrojuglone glucoside : juglone ratio for the first 4 weeks after budbreak was highest for 'Kiowa' at 6900:1 and lowest for 'Western Schley' and 'Cherokee' at 100:1 (Table 1). Although 'Kiowa' has scab resistance or immunity and 'Western Schley' and 'Cherokee' are susceptible, a high hydrojuglone glucoside: juglone ratio for resistant or immune vs. susceptible cultivars was not consistent. The hydrojuglone

Table 2. Juglone concentration (\% leaf fresh weight $) \times 10^{-3}$ in leaves of 16 pecan cultivars following the period of scab susceptibility.

\begin{tabular}{|c|c|c|c|c|c|c|c|c|c|c|}
\hline \multirow[b]{2}{*}{ Cultivar } & \multirow{2}{*}{$\begin{array}{l}\text { Scab } \\
\text { rating }^{\mathrm{y}}\end{array}$} & \multicolumn{8}{|c|}{ Weeks after budbreak ${ }^{\mathrm{Z}}$} & \multirow[b]{2}{*}{24} \\
\hline & & 5 & 6 & 7 & 8 & 10 & 12 & 16 & 20 & \\
\hline Barton & 1.00 & 9.0 & 3.3 & 1.5 & 0.2 & 1.4 & 2.0 & 1.1 & --- & 13.5 \\
\hline Elliott & 1.00 & ${ }_{-}^{c}$ & 5.6 & 2.7 & 17.9 & 2.1 & --- & --- & --- & --- \\
\hline Sumner & 1.00 & 0.9 & 3.6 & 4.0 & 2.6 & 0.1 & 1.4 & 5.5 & 2.6 & 13.3 \\
\hline Kiowa & 1.00 & 1.2 & 3.0 & 0.7 & 0.7 & --- & 0.3 & 26.3 & 2.0 & 3.9 \\
\hline Farley & 1.55 & 1.7 & 0.3 & --- & --- & --- & --- & 11.9 & 7.0 & 22.3 \\
\hline Stuart & 1.95 & 10.4 & 7.6 & 12.5 & 1.1 & 5.1 & --- & --- & --- & 12.7 \\
\hline Pawnee & 2.00 & --- & --- & --- & --- & --- & 1.1 & --- & 2.4 & --- \\
\hline Curtis & 2.10 & 8.3 & 0.4 & 1.3 & --- & 1.4 & --- & --- & --- & 8.7 \\
\hline Cape Fear & 2.20 & 0.8 & 5.4 & 1.1 & 0.1 & 0.2 & 0.2 & --- & 3.1 & 0.6 \\
\hline Moore & 2.65 & --- & 1.6 & 0.8 & 2.1 & 0.5 & --- & 6.8 & 5.8 & 50.4 \\
\hline Cheyenne & 2.85 & 2.0 & 8.3 & 6.6 & 3.8 & 5.4 & 0.2 & 3.7 & --- & 17.9 \\
\hline Desirable & 2.96 & 0.5 & 1.4 & --- & --- & --- & --- & --- & --- & 35.8 \\
\hline Schley & 2.98 & 3.9 & 7.9 & 1.8 & --- & 2.7 & 5.7 & 15.8 & 9.1 & 24.5 \\
\hline Western Schley & 3.70 & 4.9 & 15.3 & 11.6 & 8.6 & 4.6 & 1.7 & 2.6 & 12.7 & 13.0 \\
\hline Cherokee & 3.85 & 6.9 & 12.7 & 73.7 & 6.2 & 42.2 & 7.3 & 115.8 & 26.5 & 24.8 \\
\hline Wichita & 4.00 & 20.4 & 15.3 & 18.4 & 8.1 & 10.5 & 5.0 & 9.2 & --- & 2. \\
\hline
\end{tabular}

${ }^{\mathrm{z}}$ Mean juglone concentration for 5 to 24 weeks after budbreak.

${ }^{\mathrm{y}} 1$ = low susceptibility, $4=$ high susceptibility.

xJuglone was not detected. 


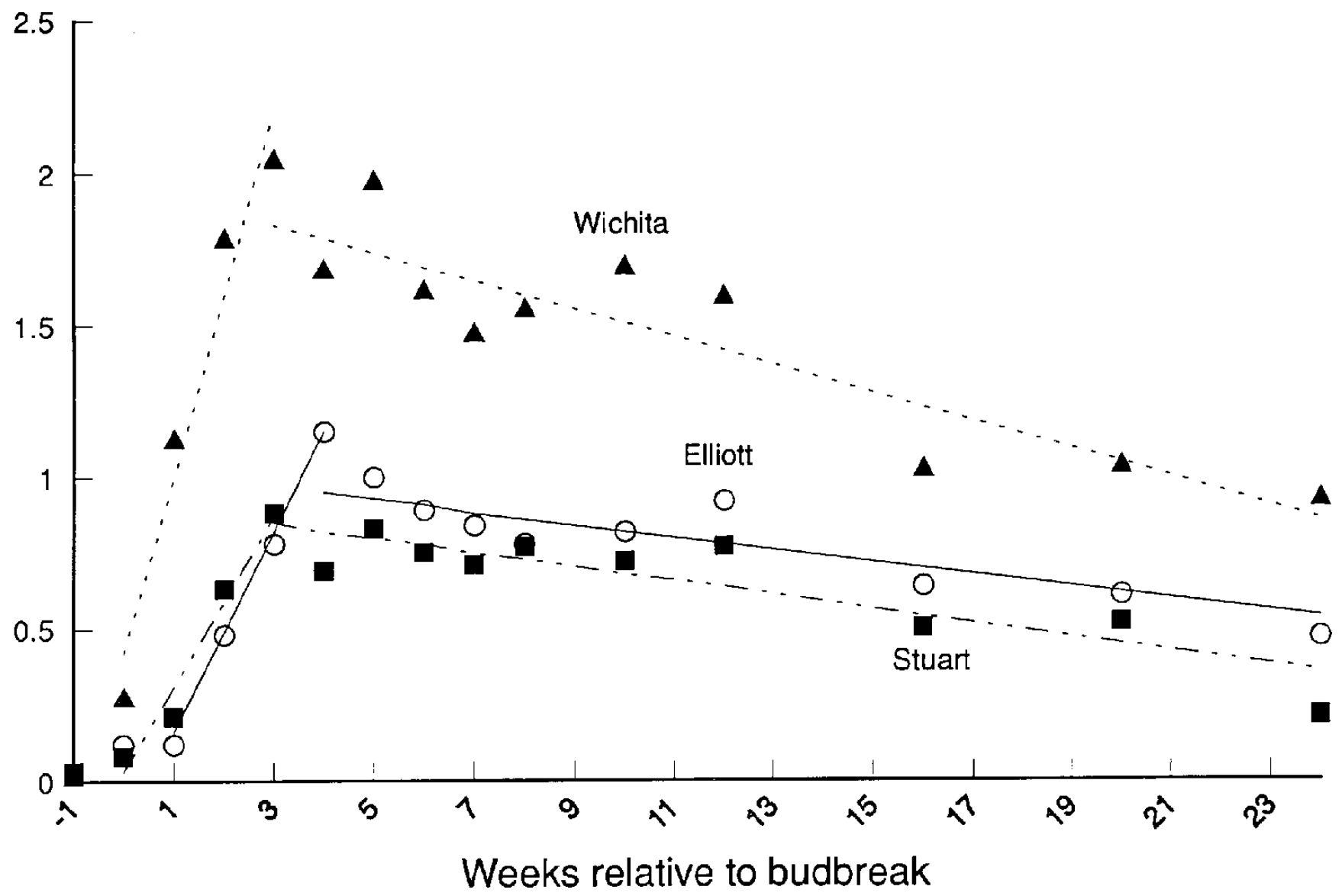

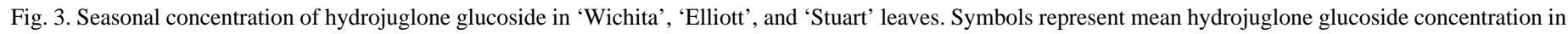

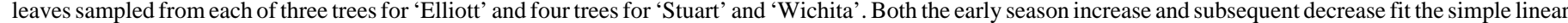

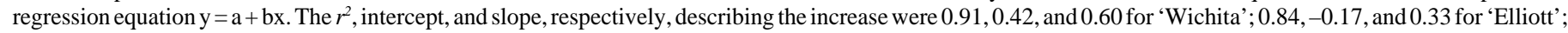

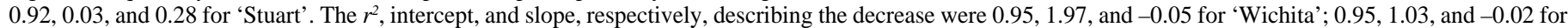
'Elliott'; 0.95, 0.92, and -0.02 for 'Stuart'; $P \leq 0.01$.

glucoside : juglone ratio for one of the least susceptible cultivars, Sumner, was only 300:1, and the ratio for the moderately susceptible cultivar, Schley, was 200:1.

The pattern of seasonal fluctuations in the hydrojuglone glucoside concentration was similar for 'Wichita', 'Elliott', and 'Stuart', with high, low, and intermediate susceptibility to Cladosprium caryigenum, respectively (Fig. 3). The hydrojuglone glucoside level was lowest at budbreak. A rapid, linear increase in hydrojuglone glucoside leaf concentration occurred during rapid leaf expansion 3 to 4 weeks after budbreak. Once leaves were at or near maximum expansion, hydrojuglone glucoside declined gradually, but never decreased to budbreak levels.

Abundance of hydrojuglone glucoside in cultivars with varying levels of scab susceptibility. A correlation did not exist between cultivar scab susceptibility and hydrojuglone glucoside concentration in the leaf either at 2,4 , or 8 weeks after budbreak $\left(r^{2}=0.34\right.$, 0.26 , and 0.30, respectively) (Fig. 4). Hydrojuglone glucoside levels were highest in three of the most susceptible cultivars, 'Wichita', 'Cherokee', and 'Western Schley,' whether examined at 2, 4, or 8 weeks after budbreak. 'Pawnee' and 'Desirable,' with scab ratings of 2.0 and 3.0, respectively, were among the cultivars with lowest hydrojuglone glucoside concentration at all three time periods. 'Elliott', 'Sumner', and 'Kiowa', all with scab ratings of 1.0, had hydrojuglone glucoside levels simlar to the much more susceptible 'Schley' at 2 and 8 weeks after budbreak.

Abundance of juglone and hydrojuglone glucoside in leaf discs with and without scab lesions. 'Wichita' leaf discs with scab lesions had $\approx 20 \%$ more hydrojuglone glucoside and $\approx 50 \%$ more juglone than discs without visible lesions (Fig. 5). Both polyphenolic compounds in the scab-free tissue were more than double that in 'Wichita' leaves with little or no scab infection collected for the study (Figs. 3 and 4). However, the hydrojuglone glucoside concentration was still higher than juglone even in scab-infected tissue.

\section{Discussion}

Hydrojuglone glucoside was first isolated and identified from Juglandaceae by Daglish (1950), who reported that the sugar moiety was attached to the 5-hydroxyl. However, the analytical protocol used by Daglish (1950) was not specific enough to accurately define where the glucose was attached. In 1955, Hayes and Thomson demonstrated conclusively that glucose was attached to the 4-hydroxyl. Thus, the proper chemical nomenclature would be 4-8-dihydroxy-1-naphthyl $\beta$ - $D$-glucopyranoside.

Two conflicting hypotheses exist on the biosynthetic pathway and existence of juglone and hydrojuglone glucoside in plants of the Juglandaceae. Daglish (1950) determined that hydrojuglone glucoside existed in vivo in plants and attributed earlier reports of free juglone in vivo to inadequate extraction techniques. $\mathrm{He}$ proposed that hydrojuglone glucoside was hydrolyzed to yield 1,4,5-trihydroxy-naphthalene, which then was oxidized to form free juglone during the extraction process. In contrast, Müller and Leistner (1978) proposed that both juglone and hydrojuglone 


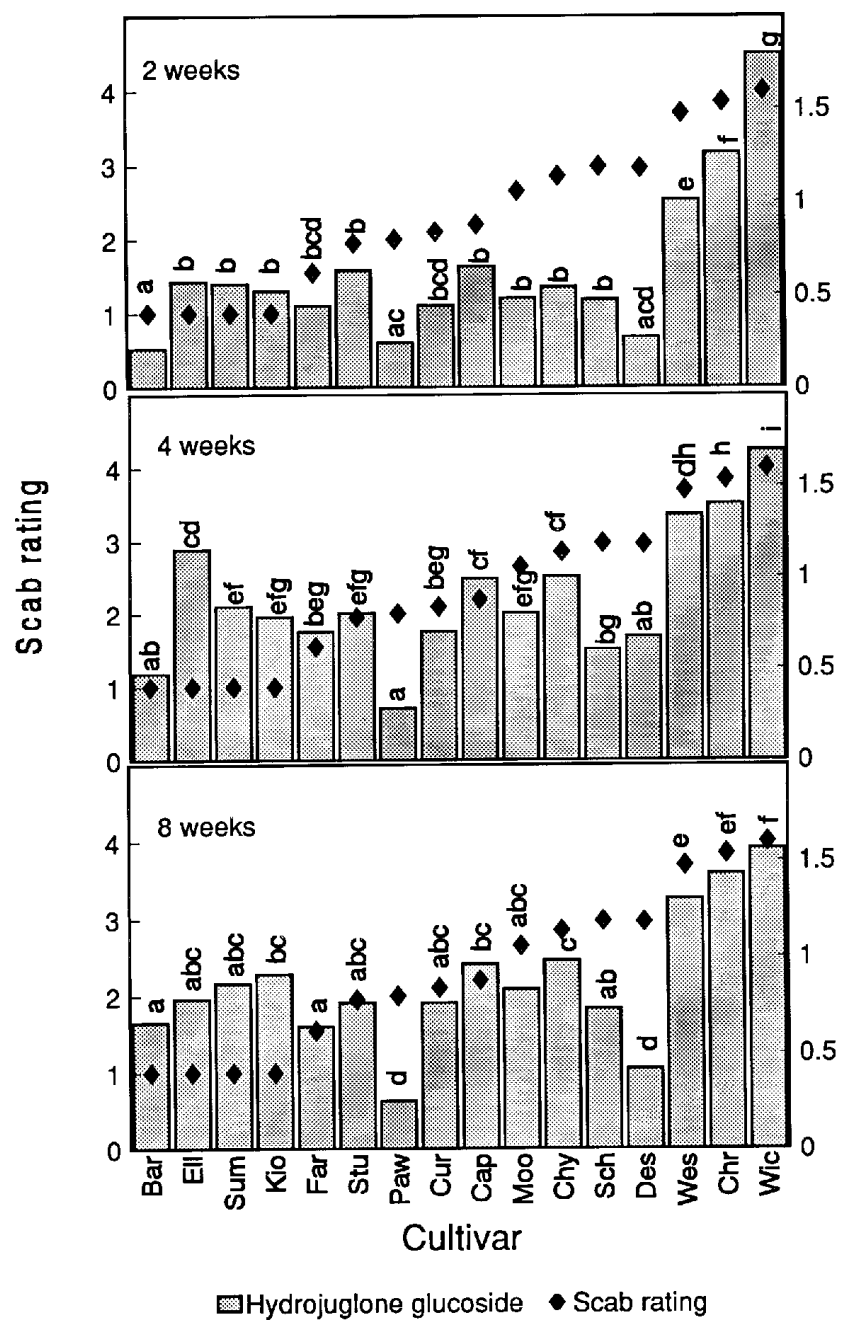

Fig. 4. Hydrojuglone glucoside in leaves of 16 pecan cultivars at 2, 4, and 8 weeks after budbreak. Cultivars with dfifferent letter are statistically different for concentration of hydrojuglone glucoside; $\mathrm{P} \leq 0.05$. Abbreviations for cultivars: Bar = 'Barton', Ell $=$ 'Elliott', Sum $=$ 'Sumner', Kio $=$ 'Kiowa', Far $=$ 'Farley', Stu = 'Stuart', Paw $=$ 'Pawnee', Cur $=$ 'Curtis', Cap $=$ 'Cape Fear', Moo = 'Moore', Chy = 'Cheyenne', Sch = 'Schley', Des $=$ 'Desirable', Wes $=$ 'Westem Schley', $\mathrm{Chr}=$ 'Cherokee', Wic $=$ 'Wichita'.

glucoside exist in vivo as metabolic end products. In the pathway proposed by Müller and Leistner (1978), hydrojuglone glucoside and juglone are formed directly from the trihydroxy-naphthalene. These investigators were convinced that juglone is a true natural product and not derived by hydrolysis of the glucoside during isolation. Harborne (1982) described the pathway as following the scheme of Daglish (1950) with hydrojuglone glucoside being the natural product. Leaching of hydrojuglone glucoside from the plant resulted in the hydrolysis and oxidation to form juglone.

Our results demonstrate that hydrojuglone glucoside, not juglone, is the major polyphenolic constituent of pecan leaves in vivo. These results are more consistent with previous documentation in walnut (Cline and Neely, 1984; Daglish, 1950) than in pecan (Borazjani et al., 1985; Diehl et al., 1992; Hedin et al., 1979). Methodology may account for the disparate results of earlier and current studies with pecan. In a study by Hedin et al. (1979), leaf samples were chilled immediately on collection and stored at $-10 \mathrm{C}$ for an unspecified time period until ground in an extraction medium of 2 chloroform : 1 methanol. Walnut tissue samples stored at $-10 \mathrm{C}$ showed a significant loss of hydrojuglone glucoside over a 48- to 72-h period (Cline and Neely, 1984). In our study,

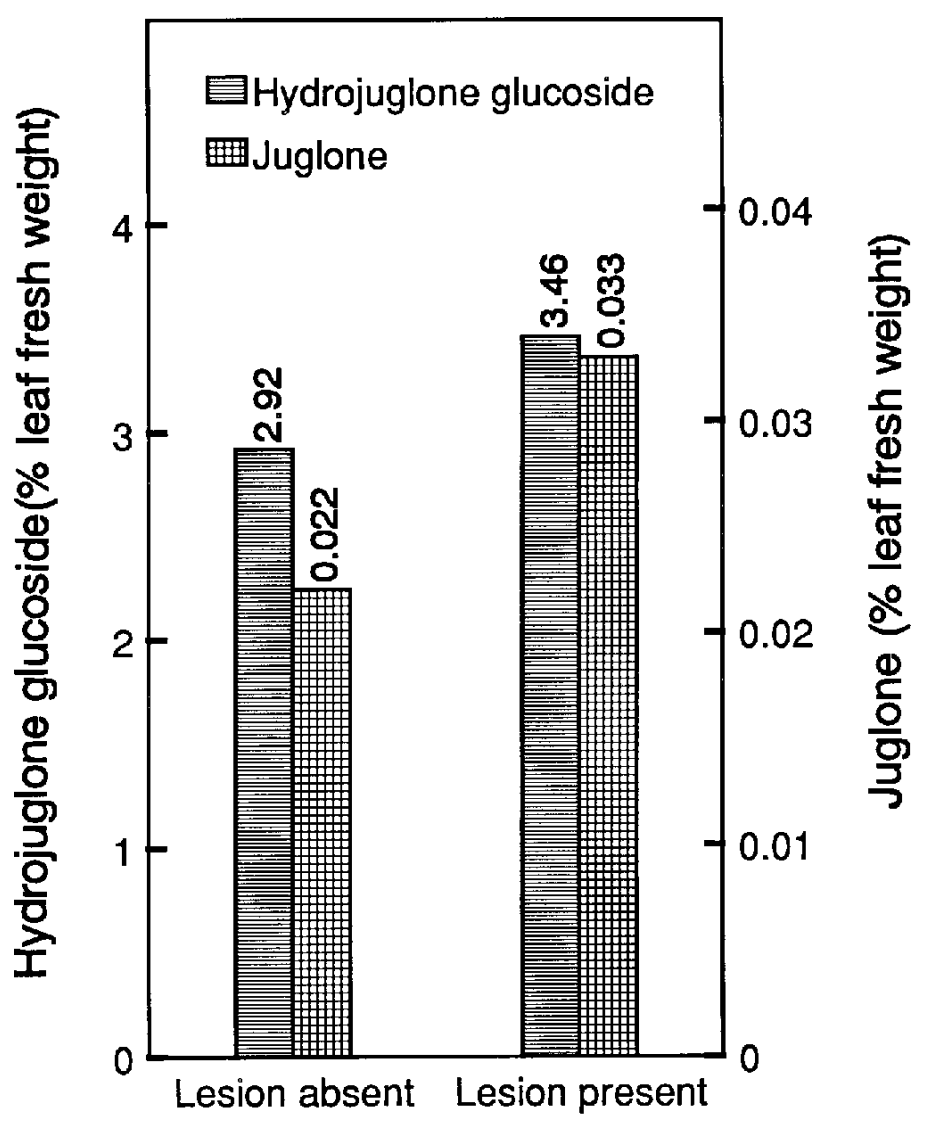

\section{Wichita leaf tissue}

Fig. 5. Hydrojuglone glucoside and juglone in 'Wichita' leaf tissue in relation to the absence or presence of scab lesions.

small quantities of fresh leaf samples of pecan were homogenized at collection time in methanol alone. The storage of pecan tissues at $-10 \mathrm{C}$ for unspecified time periods before extraction may have provided conditions for hydrojuglone glucoside breakdown. Furthermore, Hedin et al. (1979) analyzed leaf extracts by TLC and scraped off the visible yellow-orange band at $R_{f} 0.40$. Consequently, hydrojuglone glucoside may have been excluded by a decreased solubility during extraction in chloroform : methanol and/or a lower $\mathrm{R}_{\mathrm{f}}$ than juglone during fractionation and selection on TLC.

Hydrojuglone glucoside and juglone have been associated with disease resistance in members of the Juglandaceae, the plant family to which Juglans sp. (walnut) and Carya sp. (hickory and pecan) belong. The higher juglone concentration in immature than in mature walnut leaves has been proposed as a resistance mechanism in immature leaves to anthracnose caused by Gnomonia leptostyla (Cline and Neely, 1984). Speculation on juglone's role in scab resistance of hickories and pecan has developed from reported correlations involving juglone toxicity to Cladosporium caryigenum (Hedin et al., 1979); distribution among plant organs (Borazjani et al., 1985); and concentration among genotypes and during the growing season (Hedin et al., 1979). However, juglone's capacity to confer scab resistance is doubtful considering that concentrations of $10^{-3} \mathrm{M}$ juglone did not inhibit colony growth (Wood et al.,1988). Furthermore, in our study, juglone was detected only sporadically at low levels in leaves from resistant cultivars during the first 28 days after budbreak when juglone would be required to confer the scab resistance. 
Developing pecan leaves, analyzed by HPLC, demonstrated that hydrojuglone glucoside increased rapidly from budbreak until completion of leaf expansion and decreased slowly during the rest of the growing season. Juglone was detectable only in trace amounts, if at all, in both immature and mature pecan leaves. Detectable juglone accumulation was sporadic within and among cultivars and was not accounted for by genotypic resistance or scab immunity. No correlation was detected among cultivars with scab resistance or immunity and the abundance of either hydrojuglone glucoside or juglone in the 16 cultivars.

Leaf discs with scab lesions had more hydrojuglone glucoside and juglone than scab-free discs. It is unknown whether the increased levels of these polyphenolic compounds are specific responses for pecan tissue infection by Cladosporium caryigenum or general responses generated as a result of pecan tissue injury. Plant responses to mechanical injury and pathogenic infection are often similar (Bostock and Stermer, 1989). In the Harborne (1982) scenario, pecan leaf cells wounded either by insects, pathogens, or mechanical injury would be expected to activate or release enzymes needed to convert hydrojuglone glucoside to juglone.

In summary, in vivo levels of neither hydrojuglone glucoside nor juglone seem to account for cultivar genotypic susceptibility to scab disease of healthy pecan leaves. However, differences in mechanisms of sequestering hydrojuglone glucoside or factors associated with conversion of hydrojuglone glucoside to juglone and the location of this juglone possibly may be a factor. Characterization and localization of cellular events associated with the growth inhibition of Cladosporium caryigenum could be helpful in identifying factors associated with a resistance response.

\section{Literature Cited}

Anonymous, 1970. Dooryard pecan trees in Louisiana. Circ. Lflt. to Homeowner. U.S. Dept. of Agr. Pecan Lab., Shreveport, La.

Arrendale, R.F., R.F. Severson, and O.T. Chortyk. 1984. Open split interface for capillary gas chromatography/mass spectrometry. Anal. Chem. 56:1533-1537.

Bell, E.A. 1981. The physiological role(s) of secondary (natural) products, p. 1-19. In: P.K. Stumpf and E.E. Conn (eds.). The biochemistry of plants-A comprehensive treatise. vol. 7. Secondary plant products. Academic Press, New York.

Borazjani, A., C.H. Graves, Jr., and P.A. Hedin. 1985. Occurrence of juglone in various tissues of pecan and related species. Phytopathology 75:1419-1421.

Bostock, R.M. and B.A. Stermer. 1989. Perspectives on wound healing in resistance to pathogens. Annu. Rev. Phytopathol. 27:343-371.

Cline, S. and D. Neely. 1984. Relationship between juvenile-leaf resistance to anthracnose and the presence of juglone and hydrojuglone glucoside in black walnut. Phytopathology 74:185-188.

Daglish, C. 1950. The isolation and identification of a hydrojuglone glycoside occurring in the walnut. Biochem. J. 47:452-457.

Demaree, J.B. 1924. Pecan scab with special reference to sources of the early spring infections. J. Agr. Res. 28:321-333.

Diehl, S.V., C.H. Graves, Jr., and P.A. Hedin. 1992. Cytochemical responses of pecan to Cladosporium caryigenum: Development of specific histological indicators to identify and analyze in situ fungitoxic phenols. Phytopathology 82:1033-1036.

Ellis, H.C., P. Bertrand, T.F. Crocker, and S. Brown. 1991. 1992 Georgia pecan spray guide. Univ. Ga. Coop. Ext. Bul. 841.

Gomez, K.A. and A.A. Gomez. 1984. Statistical procedures for agricultural research. Wiley, New York.

Harborne, J.B. 1982. Introduction to ecological biochemistry. Academic, New York.

Hayes, N.F. and R.H. Thomson. 1955. The structure of hydrojuglone glucoside. J. Chem. Soc. 904-907.

Hedin, P.A., V.E. Langhans, and C.H. Graves, Jr. 1979. Identification of juglone in pecan as a possible factor of resistance to Fusicladium effusum. J. Agr. Food Chem. 27:92-94.

Langhans, V.E., P.A. Hedin, and C.H. Graves, Jr.. 1978. Fungitoxic chemicals in pecan tissue. Plant Dis. Rpt. 62:894-898.

Latham, A.J. and A.E. Rushing. 1988. Development of Cladosporium caryigenum in pecan leaves. Phytopathology 78:1104-1108.

MGller, W.-U. and E. Leistner. 1978. Metabolic relation between naphthalene derivatives in Juglans. Phytochemistry 17:1735-1738.

Nolen, R.E. 1926. Pecan scab. Univ. Fla. Agr. Expt. Sta. Bul. 181. Gainesville.

Romberg, L.D. 1931. Most desirable varieties for Texas, and reasons. Proc. Natl. Pecan Assn. Bul. 30:35-43.

Sanderlin, R.S. 1987. Evaluation of pecan cultivars and USDA selections for scab disease susceptibility. Res. Rpt., Pecan Res. Expt. Sta., La. Agr. Expt. Sta., Shreveport.

Sherman, W. and N. Gammon. 1977. Pecan performance in north central Florida. Pecan South 4(1):38-40.

Sparks, D. 1992. Pecan cultivars-The orchard's foundation. Pecan Production Innovations, Watkinsville, Ga.

Thompson, T.E. and L.J. Grauke. 1990. Pecans and hickories (Carya). Acta Hort. 18:839-904.

Wood, B.W., T.R. Gottwald, and C.C. Reilly. 1988. Pecan phylloplane chemicals influence germination of pecan scab conidia. J. Amer. Soc. Hort. Sci. 113:616-619.

Wood, B.W., J.A. Payne, and L.J. Grauke. 1990. The rise of the U.S. pecan industry. HortScience 25:594, 721-723. 